\title{
The influence of fan system and air conditioner on indoor particles in university dormitories
}

\author{
Zhaoyu Wang ${ }^{1, a}$, Chunjia Huang ${ }^{2, b}$ and Guozhong Zheng ${ }^{2, c, *}$ \\ ${ }^{1}$ Department of Electric Power Engineering ,North China Electric Power University, Baoding, China \\ ${ }^{2}$ Department of Power Engineering ,North China Electric Power University, Baoding, China \\ a email: 15176253208@163.com, bemail:15733229269@163.com \\ ${ }^{c}$ email:ansystem@126.com \\ *Corresponding author
}

Keywords: fans, air conditioner, indoor, particles.

\begin{abstract}
To get a more comfortable working and living environment, air conditioners and fans are widely used indoor. Considering that indoor air quality is closely related to people's physical and mental health, a university dormitory is selected to measure indoor particles concentration. The possible relationship between the concentration of particles and the use of air conditioner or fan are explored. The result of this study indicates that, the mass concentration of PM2.5 indoor has a function relationship with the time. This article not only describes the changing regulation of particle's mass concentration, but also compares the differences of the changing regulation of particles caused by fan system or air conditioner.
\end{abstract}

\section{Introduction}

People spend nearly 2/3 of the time indoor a day [1]. The quality of indoor air environment seriously affects people's physical and mental health [2, 3]. In order to get a more comfortable working and living environment, air conditioners and fans are generally used indoor. CCTV international site survey has showed that more than $1 / 5$ people stay in fans or air conditioners environment more than eight hours a day [4]. A university dormitory is selected to measure indoor particles concentration in the environment with fans or air conditioners. The possible relationship between the concentration of particles and the use of air conditioner or fan are explored in this paper.

\section{Experiments and Methods}

Experiment subject.The object of this experiment is a room on the 2nd floor in a dormitory. There is a window, a door, six beds and tables (the bed is above the table), an air conditioner, and a fan in it. The indoor arrangement is shown as Fig.1.

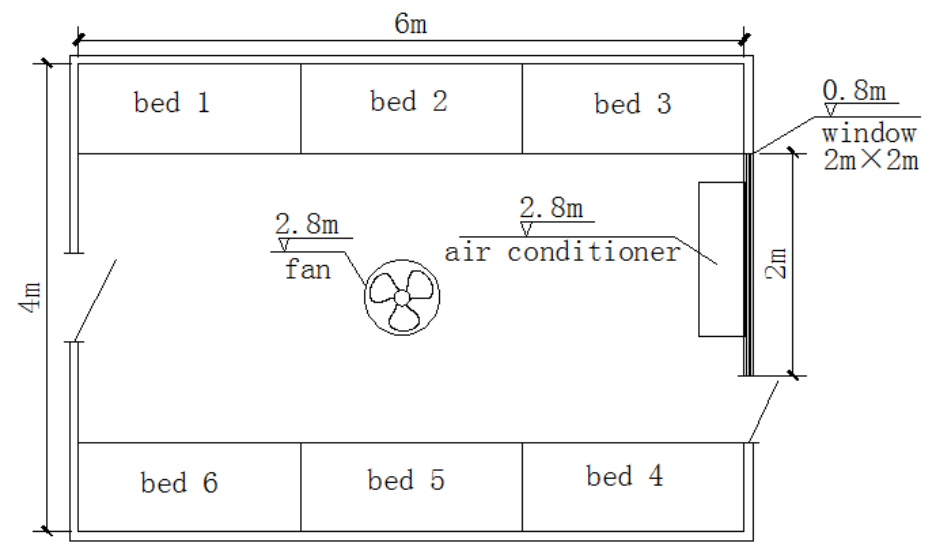

Fig.1 Indoor arrangement 
Experiment instruments. A smart dust detector (DUSTTRAK 8520) was used to measure the mass concentrations of PM2.5 and PM10.0.The number concentrations of the indoor particles were measured by a laser particle counter (AEROTRAK 8220). The experimental instruments were placed in the center of the room and the height was $0.8 \mathrm{~m}$ above the floor

Experimental scheme. The experiment includes two conditions: (1) running the fan system seperately; (2) running the air conditioner separately. The experiment of each condition lasts for 120 minutes. Every data is measured every 5 minutes until an hour later. For the fan condition, the fan is working in the largest. For the air condition condition, the temperature of the air conditioner is set at $26^{\circ} \mathrm{C}$ during every condition.

\section{Results and Discussion}

\section{Concentration characteristics of indoor particles under the fan condition}

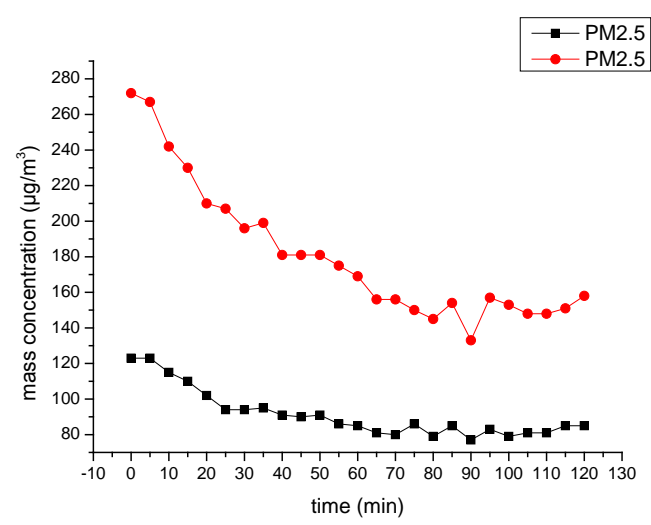

Fig.2 The dynamic changes of mass concentration

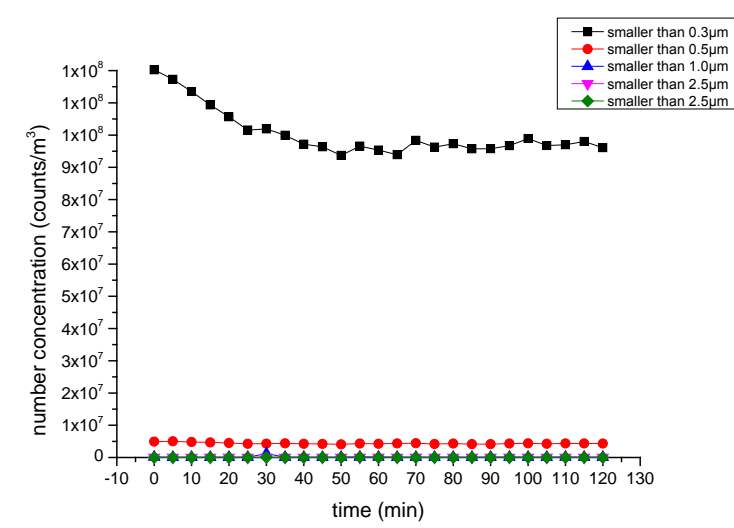

Fig.3 The dynamic changes of number of PM2.5 and PM10 under the fan concentration of PM2.5 and PM10 under the fan

Fig. 2 shows the mass concentration of particles. It can be seen that the mass concentration of PM10 changes relatively flat. However, the change of PM 2.5 mass concentration is volatile with time. Fig. 3 illustrates the number concentrations of PM that particle diameter $>0.3 \mu \mathrm{m}$ is much higher than those of the other particles. It can be inferred that when the fan is working, the smaller the particle diameter is, the more violent the airflow are. The number of particles with size of $0.3-1.0 \mu \mathrm{m}$ takes a great proportion in the air, but not the mass. After turning on a fan for 5 minutes, the mass concentrations of particles reaches maximum. After that, the mass concentrations gradually decrease, finally, stay in a steady state. When the fan is closed, all the mass concentration and number concentration of particle is basically in stable trend.

From above, it can make some inferences. Turning on a fan in 5 minutes, the airflow indoor is accelerated, the movements of particles flow faster, due to electrostatic interactions and gravity, the particles in the ground or on the object's surfaces turn the equilibrium state into a dynamic station, making the high concentration of particle in a short time. After the fan works smoothly, it takes time for these particles to establish a new stable state.

\section{Concentration characteristics of indoor particles under the air conditioner condition}

The studying method of the air conditioner is same with the fan, and it can reach the same points.

It can also be seen in Fig.4, when the air conditioner began to run, the mass concentration of particles is not significantly reduced. That it need some time for air conditioner to run smoothly may account for it.

Fig. 5 shows the number concentration of particles. When the air conditioner is turned on, the air temperature indoor reduces, the air flows faster, the movements of particles accelerate. However, the impact of large size of particles is relatively small. Thus, only small size of particles change obviously. 


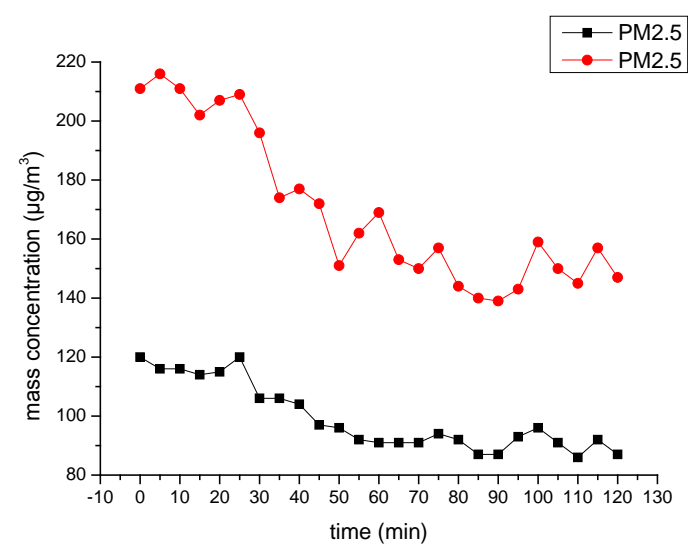

Fig. 4 The dynamic changes of mass

under the working of air conditioner

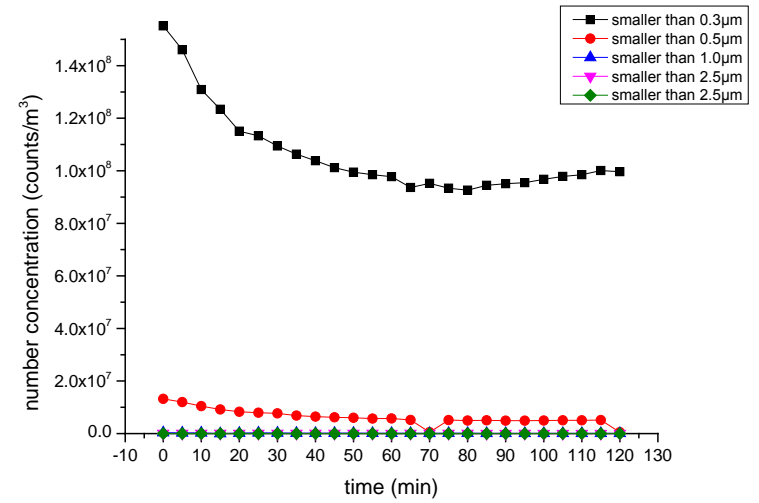

Fig. 5 The dynamic changes of number concentration concentration under the working of the air conditioner

When the air conditioner is closed, all the mass concentration and concentration of particles is basically in stable trend.

All in all, it just makes a little different in concrete value with the fan's working, accounting for the different working principles (the fan works by the machine running and reduces the temperature of skin by accelerating the air flow. The air conditioner reduces the air temperature through physics method.).

\section{Comparisons of particles under two conditions}

Table 1 Standard deviation of the mass Standard deviation of the mass concentration of PM2.5 during two hours under the working of fan

\begin{tabular}{cll}
\hline & N & Std. Deviation \\
\hline VAR00001 & 25 & 46246.3 \\
Valid N (listwise) & 25 & \\
\hline
\end{tabular}

Table.2 Standard deviation of the mass concentration of PM10 during two hours under the working of air conditioner

\begin{tabular}{lll}
\hline & N & Std. Deviation \\
\hline VAR00002 & 25 & 19579.2 \\
Valid N (listwise) & 25 & \\
\hline
\end{tabular}

In order to compare the different influences of the fan and the air conditioner, two experiments is chosen randomly and use SPSS to analyze the standard deviation of PM 2.5.

From Table 1 and Table 2, it can be concluded that running the air conditioner has few influences on indoor PM2.5 than running a fan. The possible reasons are as below:

(1) The air conditioner forms a lower airflow speed than the fan.

(2) The air conditioner reduces the indoor temperature through physical process. According to the law of gas diffusion, the higher the temperature is, the fiercer the movements of particles are. Therefore, with other factors being the same, the temperature is lower when running an air conditioner than running a fan. And the movements of PM2.5 are much slower.

\section{Summary}

This paper studies the influences of fan and air conditioner on indoor particle characteristics, and compares the different affects of between the two conditions. The conclusions are shown as follows. 
(1) The concentrations of various particles reach the maximum within 5 minutes after opening the fan, and gradually decrease over time.

(2) The conclusion of the air conditioner condition is similar to that of the fan condition. However, the air conditioner has few influences on PM2.5 when running.

(3) In order to have a better living environment, more attention need to be paid in indoor sanitation.

\section{Acknowledgments}

This paper has been supported by "Study on air pollutants of the dormitory building”(No.20142099), which belongs to the Innovation and Entrepreneurship Program for College Students in North China electric power university, and supported by "the Fundamental Research Funds for the Central Universities” (Grant No.2014QN39).

\section{References}

[1] C. Monn, Exposure assessment of air pollutants: A review on spatial heterogeneity and indoor/outdoor/personal exposure to suspended particulate matter, nitrogen dioxide and ozone, J. Atmospheric Environment. 35 (2001) 1-32.

[2] T.F. Mar, G.A. Norris, J.Q. Koenig, Associations between air pollution and mortality in Phoenix, J. Environ Health Perspect. 108 (2000)347-353.

[3] R.D. Brook, S. Rajagopalan, C.A. Pope, Particulate matter air pollution and cardiovascular disease: An update to the scientific statement from the American Heart Association, J. Circulation. 121 (2010)2331-2378.

[4] Information on http://ly.nen.com.cn/78553508734828544/20070521/1866569.shtml 